\title{
Myocardial Metabolic Abnormality in a Primary Left Atrial Rhabdomyosarcoma: Localized 1H MR Spectroscopy
}

\author{
Tae Hoon Kim, 'Gwang Woo Jeong,,$^{1,2 *}$ Tae Su Kim, ${ }^{2}$ and Yun Hyeon Kim ${ }^{2}$ \\ ${ }^{1}$ Research Institute of Medical Imaging, Chonnam National University Medical School, Gwangju, Republic of Korea \\ ${ }^{2}$ Department of Radiology, Chonnam National University Hospital, Chonnam National University Medical School, Gwangju, Republic of Korea \\ ${ }^{*}$ Corresponding author: Gwang Woo Jeong, Department of Radiology, Chonnam National University Hospital, Chonnam National University Medical School, Gwangju, Republic of Korea. \\ Tel:+82-622205881, Fax:+82-622264380, E-mail: gwjeong@jnu.ac.kr
}

Received: January 21, 2014; Revised: March 3, 2014; Accepted: March 10, 2014

\begin{abstract}
We report a 73-year-old woman with primary left atrial rhabdomyosarcoma and its $1 \mathrm{H}$ MR spectroscopy (MRS) findings. The tumor showed a $2.8 \mathrm{~cm}$ sized lesion in the posteroinferior atrial wall on MRI. 1H MR spectra were acquired using a point-resolved spectroscopy (PRESS) sequence with electrocardiographic (ECG) gating and respiratory motion. The use of $1 \mathrm{H}-\mathrm{MRS}$ allowed the quantification of triglyceride (TG) peak groups at 0.9 and $1.3 \mathrm{ppm}$, and unsaturated group of lipids at $2.1 \mathrm{ppm}$, creatine (Cr) at $3.0 \mathrm{ppm}$, and choline (Cho) at $3.2 \mathrm{ppm}$. The percentages of the myocardial metabolites based on water-peak in the interventricular septum were TG 18.4\%, $\mathrm{Cr}$ 1.6\%, Cho 3.3\% and unsaturated group 4.0\%, whereas the rhabdomyosarcoma showed TG $118.8 \%$, unsaturated group $5.1 \%$, $\mathrm{Cr} 1.3 \%$, Cho $3.5 \%$ and the olefinic components of fatty acid at $5.4 \mathrm{ppm} 24.5 \%$. This case demonstrates that $1 \mathrm{H}-\mathrm{MRS}$ is potentially useful to diagnose the rhabdomyosarcoma by quantifying the myocardial metabolites which are important biomarkers for heart function and diseases.
\end{abstract}

Keywords: Spectroscopy; Ventricular Septum; Rhabdomyosarcoma

\section{Introduction}

The first magnetic resonance spectroscopy (MRS) for the human myocardium was performed in the middle of 1980s using 31P MRS. 31P MRS requires additional hardware and software such as nucleus-specific coils (e.g. 31P coil), a broadband radiofrequency transmitter, spectroscopy pulse sequence and post-processing program (1). Furthermore, clinical use of 31P MRS technique is limited because of its inherent low sensitivity and long T1 of the metabolites, which necessitates longer data acquisition time than $1 \mathrm{H}$ MRS. Therefore, $1 \mathrm{H}$ MRS has greater potential for routine clinical applications compared with 31P MRS. With the use of $1 \mathrm{H}$ MRS of the human myocardium, the levels of myocardial metabolites such as creatine (Cr), choline (Cho), lactate (Lac) and lipids (Lip) can be quantified providing an insight into the pathophysiology of the heart diseases including myocardial infarction, heart failure, dilated or hypertrophic cardiomyopathies and cardiac tumors. Recent studies with in vivo MRS have mainly focused on myocardial infarction (2), heart failure (3), and cardiomyopathies (4), and no study has been reported about cardiac tumor. This assessment was performed to evaluate the MR spectral patterns for diagnosing the primary myocardial sarcoma using localized $1 \mathrm{H}$ MRS at 3T.

\section{Case Presentation}

A 73-year-old woman complaining of dyspnea with chest pain was admitted to our hospital, and was prescreened as arrhythmia. She underwent an examination of $1 \mathrm{H}$ cardiac MR spectroscopy. This assessment protocol was approved by the Institutional Review Board. The patient submitted an informed written consent to participate in the assessment.

To delineate and characterize the cardiac mass, a chest CT scan and cardiac MRI scans were performed. For cardiac MRI scans, this assessment was performed on a 3.0 T MRI scanner (Magnetom Tim Trio; Siemens Medical Solutions, Erlangen, Germany) with a body matrix coil. The patient was in the supine position with monitoring electrocardiography (ECG) and motion of respiration, and a series of images including 2 chamber, 4 chamber, and short axis views were acquired to provide the reference anatomic images for voxel localization of MRS. In order to minimize the distance between body matrix coil and the heart, subject and matrix coil were fastened with rubber bands. Using fast gradient echo pulse sequence with parallel acquisition techniques, 2 chamber view, 4 chamber view, and short axis view images $(\mathrm{TR} / \mathrm{TE}=8.1 / 4.5 \mathrm{~ms})$ were acquired with field of view (FOV) equal to $360 \times 360 \mathrm{~mm}^{2}$, matrix size equal to $192 \times 192$, and slice thickness equal

Copyright (C) 2015, Tehran University of Medical Sciences and Iranian Society of Radiology. This is an open-access article distributed under the terms of the Creative Commons Attribution-NonCommercial 4.0 International License (http://creativecommons.org/licenses/by-nc/4.0/) which permits copy and redistribute the material just in noncommercial usages, provided the original work is properly cited. 
to $5 \mathrm{~mm}$. In order to determine the myocardial mass and volumes (5), cardiac cine MRI was acquired using true fast imaging with steady state precession (TrueFISP) pulse sequence $(\mathrm{TR} / \mathrm{TE}=3.7 / 1.5 \mathrm{~ms})$. These examinations showed a
$2.8 \mathrm{~cm}$ vascularized mass in the left atrium causing infiltration from the left posteroinferior atrial wall (Figure 1) and the pathological findings were well consistent with those features in previous case reports $(6,7)$.
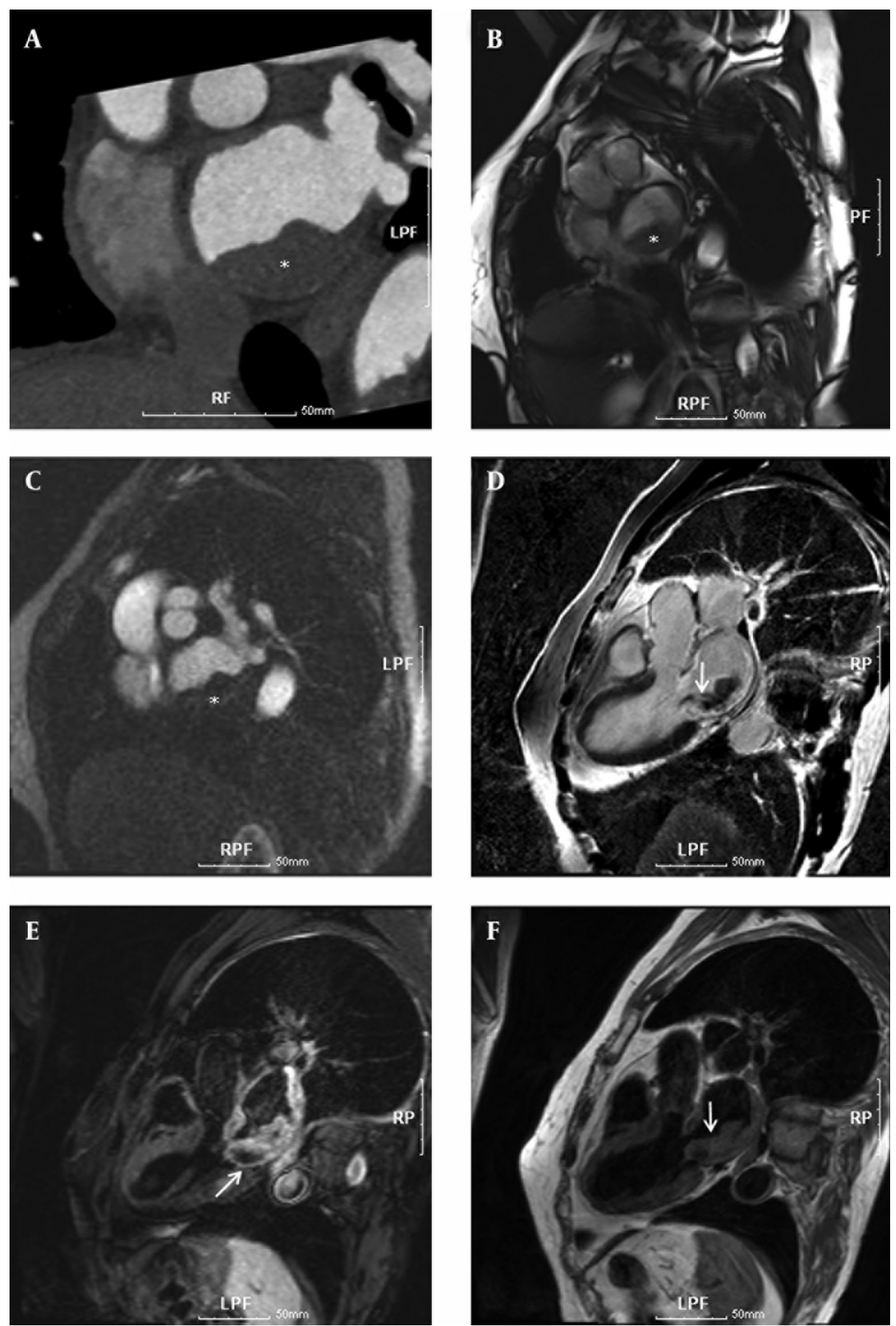

Figure 1. A 73-year-old woman with dyspnea and chest pain. Cardiac CT and MR images show a thickened mass in the left atrium, causing infiltration from the left posteroinferior atrial wall. A, Thoracic CT angiography (100 KVp, $270 \mathrm{mAs}$ ). B, True fast imaging with steady state precession pulse sequence (TR/ $\left.\mathrm{TE}=3.7 / 1.5 \mathrm{~ms}, \mathrm{FA} 50^{\circ}\right) . \mathrm{C}$, Cardiac MR perfusion image $\left(\mathrm{TR} / \mathrm{TE}=177.2 / 1.0 \mathrm{~ms}\right.$, FA $\left.10^{\circ}\right)$. D, Delayed enhanced MR image $\left(\mathrm{TR} / \mathrm{TE} / \mathrm{TI}=696 / 1.6 / 230 \mathrm{~ms}, \mathrm{FA} 20^{\circ}\right)$ at 10 minutes after injection of gadolinium-based contrast media. E, fat-suppressed T2-weighted MR image (fast spin echo; TR/TE/TI = 1743.5/49/180 ms, FA 180 ${ }^{\circ}$; and F, T1-weighted MR image with double inversion recovery (IR) pulse (spin echo; TR/TE $=1068.2 / 28 \mathrm{~ms}, \mathrm{FA} 180^{\circ}$ ). The lesion showed iso-signal intensity on double IR T1-weighted image (F), slightly higher signal intensity on the fat-suppressed T2-weighted image (E), and well enhanced signal intensity resulting from somewhat hemorrhagic necrotic change on the delayed enhanced MR images. White asterisks *, and arrows indicate the rhabdomyosarcoma 
For MR spectroscopy examination, the single-voxel 1H-MRS was performed using a point-resolved spectroscopy (PRESS) sequence with TR/TE $=2000 / 30 \mathrm{~ms}$, flip angle $=90^{\circ}, 64$ acquisitions (within 1 R-R interval; scanning time 2 minutes 34 sec- onds), $1200 \mathrm{~Hz}$ spectral width, 1024 data points, and $4.5 \mathrm{~cm}^{3}$ $\left(10 \times 15 \times 30 \mathrm{~mm}^{3}\right)$ voxel size in the interventricular septum and $6.0 \mathrm{~cm}^{3}\left(20 \times 20 \times 15 \mathrm{~mm}^{3}\right)$ in the rhabdomyosarcoma area of the posteroinferior wall of the left atrium (Figure 2)
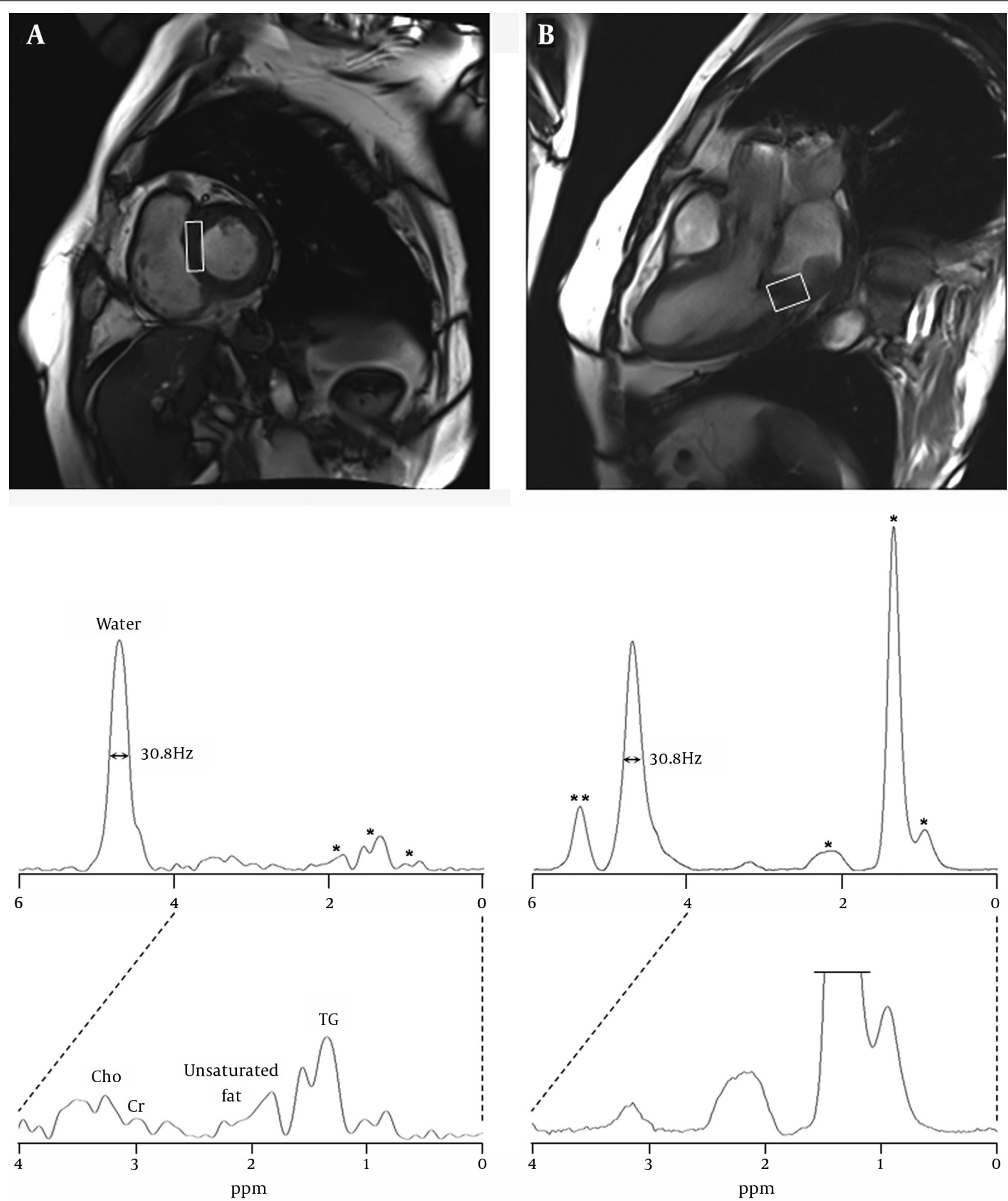

Figure 2. Cardiac MR images were acquired using true fast imaging with steady state precession (TrueFISP) pulse sequence $(\mathrm{TR} / \mathrm{TE}=3.7 / 1.5 \mathrm{~ms})$. A, shortaxis and B, four-chamber views with localized spectroscopic voxels, $10 \times 15 \times 30 \mathrm{~mm}^{3}$ in the normal interventricular septum; $20 \times 20 \times 15 \mathrm{~mm}^{3}$ in the rhabdomyosarcoma on the posteroinferior left atrial wall.1H MR spectra were acquired using PRESS sequence with TR/TE equal to 2000/30 ms and NEX equal to 64. The spectral intensities were normalized to the residual water peak demonstrating its full width at a half maximum, $30.8 \mathrm{~Hz} .{ }^{*}$ indicates triglyceride (TG) components at 0.9-2.2 ppm. ${ }^{* *}$ indicates the unsaturated groups of fatty acid at $5.4 \mathrm{ppm}$ 
Hoon Kim Tet al.

Table 1. Myocardial Metabolites in the Interventricular Septum and Rhabdomyosarcoma of the Posteroinferior Left Atrial Wall a

\begin{tabular}{lccccc}
\hline \multirow{2}{*}{ Metabolites } & \multirow{2}{*}{ Concentration, ppm } & \multicolumn{2}{c}{ Interventricular Septum } & \multicolumn{2}{c}{ Rhabdomyosarcoma } \\
\cline { 3 - 6 } & & Integral & Percentage $^{\mathrm{b}}$ & Integral & Percentage $^{\mathrm{b}}$ \\
\hline Water & $(4.70)$ & 748.51 & 100.0 & 12026.44 & 100.0 \\
Cho & $(3.20)$ & 24.99 & 3.3 & 418.32 & 3.5 \\
Cr & $(3.02)$ & 12.16 & 1.6 & 152.22 & 1.3 \\
Unsaturated fat & $(2.10)$ & 30.29 & 4.0 & 610.38 & 5.1 \\
TG & $(0.90,1.30)$ & 135.97 & 18.4 & 14297.55 & 118.8 \\
Olefinic components & $(5.40)$ & - & - & 2942.46 & 24.5 \\
\hline
\end{tabular}

a Abbreviations: Cho, choline; $\mathrm{Cr}$, creatine; TG, triglyceride.

$\mathrm{b}$ The metabolites were normalized to the residual water peak.

Prior to the MRS acquisition, manual shimming of the Bo magnetic field and optimization of the transmitter pulse power were performed. After shimming and gradient tuning, water suppression was accomplished with three $50 \mathrm{~Hz}$ bandwidth chemical-shift-selective saturation (CHESS) pulses, followed by spoiling gradients at the beginning of data acquisition. The raw data of MRS were post-processed and analyzed by using an MR spectroscopy data analysis package (Siemens Medical Solutions) $(8,9)$ : the free induction decay data were multiplied by Gaussian line broadening function with $5 \mathrm{~Hz}$, zerofilling ( 2 times, giving 4096 data points), and Fouriertransformed to frequency domain. The spectral baseline was corrected with nine-polynomial fitting, followed by phase correction with the zero- and first-order. The residual water peak at $4.7 \mathrm{ppm}$ was used as an internal reference for the quantification of the metabolites including $\mathrm{Cr}$, Cho and TG (10). The percentage of myocardial metabolites relative to water was calculated as the integrals of each metabolite (11).

Resonance frequencies for intramyocellular lipids were estimated with the assumption of Gaussian line shapes at $0.9 \mathrm{ppm}, 1.3 \mathrm{ppm}$ and $2.1 \mathrm{ppm}$. The integrals of lipid resonances at 0.9 and $1.3 \mathrm{ppm}$ were summed for TG quantification. Also, prior knowledge for the chemical shift of metabolites was incorporated into the fitting algorithm by using previous criteria (11-13). For analysis of MR spectra, major myocardial metabolites were assigned as follows: choline (Cho, $3.20 \mathrm{ppm})$, creatine (Cr, $3.02 \mathrm{ppm})$, unsaturated group of lipids (2.10 ppm) and triglyceride (TG, 0.91 and $1.30 \mathrm{ppm}$ ).

The full-width at half maximum (FWHM) of both residual water peaks in the septum and the sarcoma of posteroinferior left atrial wall was $30.8 \mathrm{~Hz}$. The levels of myocardial metabolites are summarized in Table 1 . In the septum, the percentages of myocardial metabolites were $18.4 \%$ TG, $4.0 \%$ unsaturated group of lipids, $1.6 \% \mathrm{Cr}$ and 3.3\% Cho, whereas the sarcoma showed $118.8 \% \mathrm{TG}, 5.1 \%$ unsaturated group of lipids, $1.3 \% \mathrm{Cr}$ and 3.5\% Cho. Also, the olefinic components of fatty acid or cholesterol in the rhabdomyosarcoma were identified at $5.4 \mathrm{ppm}$ and its percentage was $24.5 \%$.

\section{Discussion}

Cardiac 1H-MRS with ECG gating and respiratory motion at 3T MR system was allowed to achieve quantitative information of myocardial metabolites from small voxel volumes $(<5 \mathrm{~mL})$ in the interventricular septum.

The acquisition time of a single spectrum was about 3 minutes, and the total examination time including MR spectroscopy with cardiac anatomic imaging was less than 50 minutes. With regard to spectral quality in the human myocardium, FWHM of the residual water peak was reproducible values, about $30 \mathrm{~Hz}$. Accurate and precise positioning with adequate voxel size on the sarcoma of the posteroinferior left atrial wall is essential for accurate quantification of the metabolites. The MRS voxels were localized to avoid the contamination of unwanted tissues such as perimyocardium and chest muscle and the voxel sizes were optimized to cover the focal regions with minimal sizes, septum $\left(4.5 \mathrm{~cm}^{3}\right)$ and myosarcoma $\left(6.0 \mathrm{~cm}^{3}\right)$. In quantification of the resonance peaks in spectra of the septum and myosarcoma, the metabolic signal intensities were normalized to the residual water peak by weak water suppression as shown in Figure $2(4,10,14)$.

This study was performed to compare quantitative metabolic information acquired from the normal septum and sarcoma region of the left atrial wall. The heart is the main myogenic muscular organ in the circulatory system and the spectral appearance of normal septal myocardium in this study was considerably similar to the spectral pattern in the skeletal muscle (14). Rhabdomyosarcoma is a type of sarcoma that rarely occurs in the striated muscle. The voxel size of rhabdomyosarcoma was localized within the tumor part with a maximal thickness of $2.8 \mathrm{~cm}$, and the resulting spectral pattern was similar to that of the calf muscle (voxel size of $180 \mathrm{~cm}^{3}$ ) reported in Bongers' study (15).

Cho is a marker of cell membrane metabolism/turnover and is involved in acetylcholine (ACh) synthesis with acetyl CoA and cholinergic neurotransmission. It is well known that the Cho peak is a common biomarker for cancers with a variety of histological types (16). Also, the Cho level is closely related to the degree of malignancy of 
Hoon Kim Tet al.

the hypercellular tumors with rapid growth. In the present study, however, the Cho levels in both normal and lesional myocardium were in the normal range, and the concentration difference was not significant, suggesting that the sarcoma is in the early stage of malignancy (Table 1). Myocardial $\mathrm{Cr}$ is associated with high-energy phosphate metabolism as an indicator of oxidative metabolism and the previous study showed that reduction of energy reserve underlies impaired contractile function in the failing heart (3). Consequently, the tumor-specific abnormalities of $\mathrm{Cho}$ and $\mathrm{Cr}$ metabolites were not found in this case.

On the other hand, the peak at $5.4 \mathrm{ppm}$ on the spectrum of rhabdomyosarcoma was assigned to $\mathrm{C} 6$ protons of cholesterol and/or the unsaturated groups of the olefinic region of lipids $(15,17)$. In addition, the TG metabolite at 0.9 and $1.3 \mathrm{ppm}$ showed the prominent difference, giving $18 \%$ in the septum and $119 \%$ in the sarcoma. In an animal study (18), TG metabolite has shown a negative correlation with heart function, while treatment with insulinsensitizing drugs reduced myocardial TG deposition and reversed contractile dysfunction in lipotoxic heart disease in rats with obesity. Also, myocardial TG may be a marker of myocardial viability after coronary occlusion due to enhanced esterification and/or reduced oxidation of fatty acids in ischemically insulted but viable myocardium (19). These findings suggest that intramyocelluar TG accumulation is deleterious to the heart. Therefore, $1 \mathrm{H}$ MRS is a promising tool for assessing TG metabolites in human myocardial tissue.

This study included several technical limitations. First, the signal intensities on the focal regions may contain contaminations of unwanted signal from the outside of the voxel. Second, the differential voxel sizes ( $4.5 \mathrm{cc}$ vs. $6.0 \mathrm{cc}$ ) on the normal septum and rhabdomyosarcoma may give rise to a quantitative error because of different signal-to-noise ratio. Third, this study used the residual water peak as a reference for quantification of the metabolites in the myocardium. However, the use of residual water signal can be prone to quantification error, due to cardiac motion and different water contents in different tissues. Fourth, the finding of increased TG content may not be specific to cardiac sarcoma because the predominant changes in TG content are frequently reported for various cardiac diseases and cancer. This study dealt with one patient and further studies are needed to confirm a more specific finding from a large population.

Although the present case report had several limitations, the advantages of $1 \mathrm{H}$ MRS over other cardiac imaging techniques, PET and contrast media-based MRI are that it does not expose patients to ionizing radiation and side effect of agents. Therefore, $1 \mathrm{H}$ MRS is widely accessible for assessing the normal myocardium and myocardial tumors (about 5 minutes in both tissues), and can concurrently provide the information of quantitative metabolic concentrations for effective therapeutic strategies in clinical settings.

In conclusion, this study, for the first time, demon- strates the feasibility that cardiac $1 \mathrm{H}-\mathrm{MRS}$ would be potentially useful for the diagnosis of rhabdomyosarcoma by the use of TG level in the myocardium.

\section{Authors' Contributions}

Study concept and design: Yun-Hyeon Kim and GwangWoo Jeong; Acquisition of data: Tae-Hoon Kim, Tae-Su Kim, and Yun-Hyeon Kim; Analysis and interpretation of data: Tae-Hoon Kim, Tae-Su Kim, and Gwang Woo Jeong; Drafting of the manuscript: Tae-Hoon Kim and GwangWoo Jeong; Critical revision of the manuscript for important intellectual content: Yun-Hyeon Kim and GwangWoo Jeong; Study supervision: Gwang-Woo Jeong.

\section{Financial Disclosure}

All authors declare that they have no financial interests related to the material in the manuscript.

\section{Funding/Support}

This work was supported by the Grants of the National Research Foundation of Korea (NRF) (No. 2011-0016291 and 2012R1A6A3A01017458).

\section{References}

1. Bottomley PA. Noninvasive study of high-energy phosphate metabolism in human heart by depth-resolved 31P NMR spectroscopy. Science. 1985;229(4715):769-72.

2. Bottomley PA, Wu KC, Gerstenblith G, Schulman SP, Steinberg A, Weiss RG. Reduced myocardial creatine kinase flux in human myocardial infarction: an in vivo phosphorus magnetic resonance spectroscopy study. Circulation. 2009;119(14):1918-24.

3. Lygate CA, Schneider JE, Neubauer S. Investigating cardiac energetics in heart failure. Exp Physiol. 2013;98(3):601-5.

4. Nakae I, Mitsunami K, Yoshino T, Omura T, Tsutamoto T, Matsumoto $\mathrm{T}$, et al. Clinical features of myocardial triglyceride in different types of cardiomyopathy assessed by proton magnetic resonance spectroscopy: comparison with myocardial creatine. $J$ Card Fail. 2010;16(10):812-22.

5. Malayeri AA, Johnson WC, Macedo R, Bathon J, Lima JA, Bluemke DA. Cardiac cine MRI: Quantification of the relationship between fast gradient echo and steady-state free precession for determination of myocardial mass and volumes. J Magn Reson Imaging. 2008;28(1):60-6.

6. Conquest HF, Thornton JL, Massie JR, Coxe J3. Primary Pulmonary Rhabdomyosarcoma; Report of Three Cases and Literature Review. Ann Surg. 1965;161:688-92.

7. Taratuto AL, Molina HA, Diez B, Zuccaro G, Monges J. Primary rhabdomyosarcoma of brain and cerebellum. Report of four cases in infants: an immunohistochemical study. Acta Neuropathol. 1985;66(2):98-104.

8. Kim TH, Kang HK, Jeong GW. Assessment of brain metabolites change during visual sexual stimulation in healthy women using functional MR spectroscopy. J Sex Med. 2013;10(4):1001-11.

9. Kim TH, Kang HK, Park K, Jeong GW. Localized brain metabolite changes during visual sexual stimulation in postmenopausal women: a pilot study using functional magnetic resonance spectroscopy. Menopause. 2014;21(1):59-66.

10. Reyngoudt H, Claeys T, Vlerick L, Verleden S, Acou M, Deblaere $\mathrm{K}$, et al. Age-related differences in metabolites in the posterior cingulate cortex and hippocampus of normal ageing brain: $\mathrm{a} 1 \mathrm{H}-$ MRS study. EurJ Radiol. 2012;81(3):e223-31.

11. Fayad LM, Salibi N, Wang X, Machado AJ, Jacobs MA, Bluemke DA, et al. Quantification of muscle choline concentrations by proton MR spectroscopy at $3 \mathrm{~T}$ : technical feasibility. AJR Am J Roentgenol. 2010;194(1):W73-9. 
12. Boesch C, Slotboom J, Hoppeler H, Kreis R. In vivo determination of intra-myocellular lipids in human muscle by means of localized 1H-MR-spectroscopy. Magn Reson Med.1997;37(4):484-93.

13. Rico-Sanz J, Hajnal JV, Thomas EL, Mierisova S, Ala-Korpela M, Bell JD. Intracellular and extracellular skeletal muscle triglyceride metabolism during alternating intensity exercise in humans. $J$ Physiol. 1998;510 ( Pt 2):615-22.

14. Bottomley PA, Lee Y, Weiss RG. Total creatine in muscle: imag ing and quantification with proton MR spectroscopy. Radiology. 1997;204(2):403-10.

15. Bongers H, Schick F, Skalej M, Hess CF, Jung WI. Localised in vivo $1 \mathrm{H}$ spectroscopy of human bone and soft tissue tumours. European Radiology. 1992;2(5):459-464..

16. Bartella L, Morris EA, Dershaw DD, Liberman L, Thakur SB,
Moskowitz C, et al. Proton MR spectroscopy with choline peak as malignancy marker improves positive predictive value for breast cancer diagnosis: preliminary study. Radiology. 2006;239(3):686-92.

17. Wang CK, Li CW, Hsieh TJ, Chien SH, Liu GC, Tsai KB. Characterization of bone and soft-tissue tumors with in vivo $1 \mathrm{H}$ MR spectroscopy: initial results. Radiology. 2004;232(2):599-605.

18. Zhou YT, Grayburn P, Karim A, Shimabukuro M, Higa M, Baetens $D$, et al. Lipotoxic heart disease in obese rats: implications for human obesity. Proc Natl Acad Sci U S A. 2000;97(4):1784-9.

19. Straeter-Knowlen IM, Evanochko WT, den Hollander JA, Wolkowicz PE, Balschi JA, Caulfield JB, et al. 1H NMR spectroscopic imaging of myocardial triglycerides in excised dog hearts subjected to 24 hours of coronary occlusion. Circulation.1996;93(7):1464-70. 